\title{
Nanoscale
}

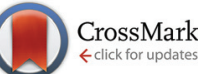

Cite this: Nanoscale, 2015, 7, 6481

Received 15th December 2014, Accepted 12th March 2015

DOI: $10.1039 / \mathrm{c} 4 \mathrm{nr} 07395 \mathrm{a}$

www.rsc.org/nanoscale

\section{Observation of polarized gain from aligned colloidal nanorods $\uparrow$}

\author{
Yuan Gao, ${ }^{\mathrm{a}, \mathrm{b}}$ Van Duong Ta, ${ }^{\mathrm{b}}$ Xin Zhao, ${ }^{\mathrm{a}, \mathrm{b}}$ Yue Wang, ${ }^{\mathrm{a}}$ Rui Chen, ${ }^{\mathrm{a}}$ \\ Evren Mutlugun, $t^{\mathrm{a}, \mathrm{b}}$ Kah Ee Fong, ${ }^{\mathrm{b}}$ Swee Tiam Tan, ${ }^{\mathrm{b}}$ Cuong Dang, ${ }^{\mathrm{b}}$ Xiao Wei Sun, ${ }^{\mathrm{b}}$ \\ Handong Sun ${ }^{\star a, b}$ and Hilmi Volkan Demir*a,b,c
}

In recent years, colloidal semiconductor nanorods have attracted great interest for polarized spontaneous emission. However, their polarized gain has not been possible to achieve so far. In this work we show the highly polarized stimulated emission from the densely packed ensembles of core-seeded nanorods in a cylindrical cavity. Here CdSe/CdS dot-in-rods were coated and aligned on the inner wall of a capillary tube, providing optical feedback for the nanorod gain medium. Results show that the polarized gain originates intrinsically from the aligned nanorods and not from the cavity and that the optical anisotropy of the nanorod ensemble was amplified with the capillary tube, resulting in highly polarized whispering gallery mode lasing. The highly polarized emission and lasing, together with easy fabrication and flexible incorporation, make this microlaser a promising candidate for important color conversion and enrichment applications including liquid crystal display backlighting and laser lighting.

Semiconductor nanocrystals are attractive materials offering numerous applications ranging from bioimaging ${ }^{1}$ to optoelectronic devices, including light emitting diodes (LEDs), ${ }^{2}$ solar cells, ${ }^{3}$ and lasers. ${ }^{4,5}$ Over the past thirty years, the development of colloidal nanocrystals has led to the achievement of high photoluminescence (PL) quantum yields with narrow emission linewidths as well as tunability of the peak emission

\footnotetext{
${ }^{a}$ Division of Physics and Applied Physics, School of Physical and Mathematical Sciences, Nanyang Technological University, 21 Nanyang Link, 637371, Singapore. E-mail: hdsun@ntu.edu.sg, hvdemir@ntu.edu.sg; Fax: +65 6316 6984; Tel: +6567905395

${ }^{b}$ LUMINOUS! Center of Excellence for Semiconductor Lighting and Displays, School of Electrical and Electronic Engineering, Nanyang Technological University, 50 Nanyang Avenue, 639785, Singapore

${ }^{c}$ Department of Electrical and Electronics Engineering and Department of Physics, UNAM - Institute of Materials Science and Nanotechnology, Bilkent University, Bilkent, Ankara, Turkey. E-mail: volkan@bilkent.edu.tr

$\dagger$ Electronic supplementary information (ESI) available: Absorption and photoluminescence spectra, calculation of anisotropy, optical and electron microscopy images, lasing characterization, and polarization response of the detectors. See DOI: $10.1039 / \mathrm{c} 4 \mathrm{nr} 07395 \mathrm{a}$

¥Department of Electrical and Electronics Engineering, Abdullah Gul University, 38039 Melikgazi, Kayseri, Turkey.
}

wavelength from the ultraviolet to the infrared by tailoring the size, structure and/or chemical composition. ${ }^{6}$ In addition, solution-based processing of these colloidal semiconductor nanoparticles has allowed for low-cost fabrication, essentially at any substrate, for diverse photonic devices.

Given the nanoscale size of semiconductor nanoparticles, their density of electronic states is atomic-like because of the quantum confinement effect, which makes the emission wavelength and optical gain of this class of material less sensitive to temperature. ${ }^{4}$ Meanwhile, their better photostability as compared to organic dyes, combined with their rare earth element free nature, makes nanocrystals promising candidates for potential applications in lasers. ${ }^{7}$

Lasing from semiconductor nanocrystals was first demonstrated in 1991 by optical pumping CdSe nanocrystal doped glass at $80 \mathrm{~K} .{ }^{8}$ However, under strong excitation that creates multiexcitons, Auger recombination becomes an undesired non-radiative channel, a competing process for stimulated emission. ${ }^{9-11}$ In order to suppress the Auger recombination, the concept of single exciton gain was introduced and realized using type-II nanocrystals where electrons and holes are distributed separately in cores and shells of the semiconductor nanocrystals. ${ }^{12}$ However, adopting type-II nanocrystals leads to an extreme reduction in the overlap of electron and hole wavefunctions so that the emission transition rate is decreased significantly. In addition, recent type-I nanocrystal lasers operating in the single exciton regime require a relatively low pumping intensity to avoid multiexciton Auger loss but this also limits their output power. ${ }^{12}$ Suppressing the Auger recombination rate, while maintaining the optical oscillation strength by engineering electron hole wave-functions, has been a subject of studies in various nanocrystal structures. ${ }^{13-16}$

A new type of semiconductor heterostructure nanocrystal in rod shape, CdSe core-seeded CdS nanorod, or CdSe/CdS dotin-rod (DR) has been recently synthesized. ${ }^{13} \mathrm{CdSe} / \mathrm{CdS}$ DRs preserve the conventional nanocrystals' discrete electronic states and exhibit a nearly temperature independent threshold. ${ }^{17}$ On the other hand, the offset of the conduction band is very small in comparison with that of the valence 
band. ${ }^{18}$ Therefore, holes are mainly confined in the CdSe core while electrons are spread in the whole CdS rod. Because of their quasi-type-II band alignment, CdSe/CdS DRs show a pronounced reduction of Auger recombination and a remarkable increase of gain lifetime. ${ }^{16,19-22}$ Given the temperature insensitive threshold and the suppression of Auger recombination, CdSe/CdS DRs are promising candidates for optical gain applications.

On the other hand, polarized light is essential to liquidcrystal display (LCD) and 3D display technologies. Polarized emission was previously observed from elongated semiconductor nanocrystals. ${ }^{23}$ As asymmetric nanocrystals with mixed dimensionality, CdSe/CdS DRs not only preserve the merits of spherical nanocrystals such as wide color tunability but also show anisotropic properties of absorption and emission. ${ }^{13,24-26}$ To utilize such anisotropic optical properties for photonic devices, CdSe/CdS DRs must be oriented in a collective way. Many methods have been designed to realize nanocrystal superlattices on a large scale to enable anisotropic emission from nanorod ensembles such as self-assembling on water surface, ${ }^{27}$ controlled evaporation, ${ }^{28}$ electric field assisted alignment, ${ }^{13,29}$ and others. However, these approaches have typically been limited to monolayers with anisotropic fluorescence and have not been able to achieve optical gain to date.

A capillary tube is a good host for a possible gain medium since it can play the role of a cylindrical optical microcavity along with the generation of whispering gallery modes (WGM) to provide optical feedback. ${ }^{30,31}$ The tubes are usually filled with optical gain solutions. However, a low refractive index contrast between the solution and the glass tube reduces the overlap of optical modes with gain medium, which makes it difficult to lase. ${ }^{30,31}$ Besides the low refractive index contrast and low density problem, randomly distributed DRs in solution cannot utilize their anisotropic dipole moment for the optical gain.

In this work, in order to address all of these outlined problems, we present the first account of the high-density packing of DRs aligned with a preferable orientation on a large scale to enable a strongly polarized gain medium. Here CdSe/CdS coreseeded nanorods were used to be aligned with the capillary tubes and cover their inner walls, forming a collective effect on the cylindrical microcavity. Spontaneous emission with a very high polarization ratio was observed to confirm the preferable orientation of the CdSe/CdS DR ensemble in the capillary tubes. Unlike WGM lasing with an isotropic gain medium in a cylindrical cavity of hundreds of micrometers in diameter, where the cavity shows negligible polarization selectivity, ${ }^{30,32}$ the WGM lasing with the polarized gain medium of these aligned CdSe/CdS DRs was strongly polarized.

CdSe/CdS DRs were synthesized using a seeded growth approach. $^{13}$ The photoluminescence quantum yield is 0.75 , determined by comparison with Rhodamine 101 in ethanol. ${ }^{33}$ The first absorption peak and the PL emission spectrum of the CdSe quantum dot and the CdSe/CdS DR solution show very well defined exciton peaks of the quantum dot structure (Fig. $\mathrm{S} 1 \dagger$ ). Absorption cross section is increased dramatically at shorter wavelengths after CdS rod growth (Fig. S1a $\dagger$ ). This helps in harvesting more pump photons and facilitates population inversion for stimulated emission. ${ }^{34}$

To verify the intrinsic anisotropy in optical properties of CdSe/CdS DRs, the fluorescence anisotropy was measured for a diluted CdSe/CdS DR solution in a cuvette. A continuous wave laser with $532 \mathrm{~nm}$ wavelength and linear polarization was used as an excitation source in the experimental setup shown in Fig. 1a. Fig. 1b presents the fluorescence spectra of a DR solution at two different polarizations, which are parallel and perpendicular to the excitation polarization, respectively. As we can see, the DR solution tends to emit photons in the same polarization as that of the excitation source. Fig. 1c shows the integrated fluorescence intensity from the DR solution at different polarization angles $(\theta)$ with respect to the excitation polarization. The fitting curve shows the $\cos ^{2} \theta$ behavior of the emission intensity. In contrast, the diluted spherical wurtzite $\mathrm{CdSeS} / \mathrm{ZnS}$ nanocrystal solution showed isotropic emission (Fig. S2a $\dagger$ ). Similar experiments were also performed with a zinc blende spherical shaped CdSe/CdS/ZnS nanocrystal solution where no anisotropic fluorescence was observed, thus ruling out the effects of a crystalline structure (Fig. S2b†). This anisotropic emission from CdSe/CdS DRs revealed the difference in dipole moment between the orientations which are along $\left(\mu_{c}\right)$ and normal $\left(\mu_{a}, \mu_{b}\right)$ to the DRs' long axis. The fluorescence anisotropy is defined as $r=\left(I_{\|}-I_{\perp}\right) /\left(I_{\|}+2 I_{\perp}\right),{ }^{29}$ where
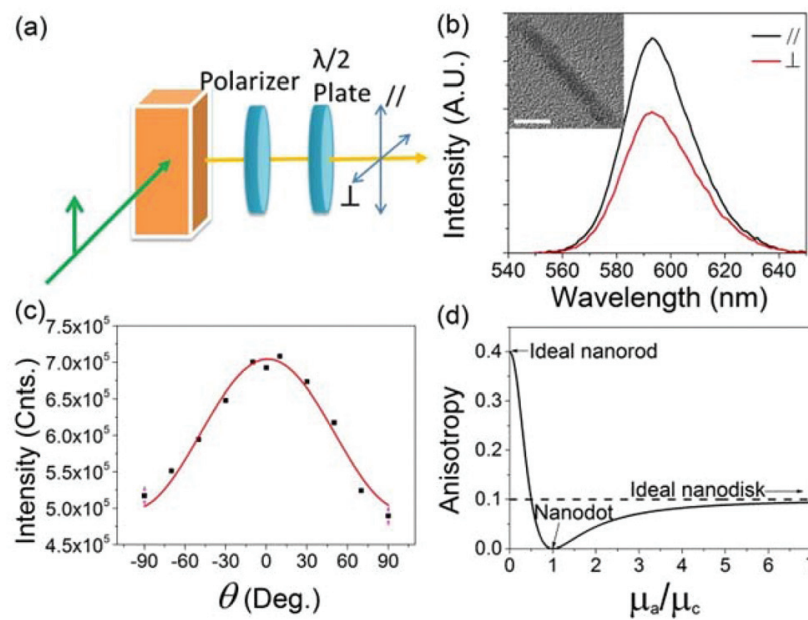

(d)

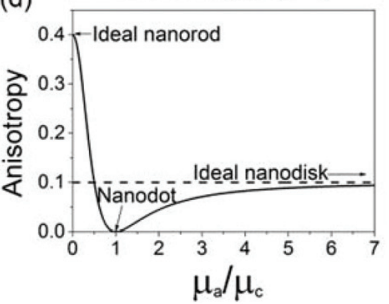

Fig. 1 Fluorescence anisotropy measurement of a diluted $\mathrm{CdSe} / \mathrm{CdS}$ DRs solution. (a) Measurement configuration for fluorescence anisotropy. The polarizer was used to select the fluorescence with the desired polarization. The achromatic half-wave plate was used to tilt the polarization of the emission horizontally before detection. (b) Spectra of emission from diluted CdSe/CdS DR solution with two different polarizations, which were parallel and perpendicular to the excitation polarization. The inset shows the high resolution transmission electron microscopy (TEM) image of a single CdSe/CdS DR (scale bar, $10 \mathrm{~nm}$ ). (c) Emission intensity of a diluted CdSe/CdS DR solution at different polarization angles with respect to the excitation polarization. (d) Fluorescence anisotropy as a function of the ratio between dipole moments for a fluorescent material with cylindrical symmetry $\left(\mu_{a}=\mu_{b} \neq \mu_{c}\right)$. 
$I_{\|}$and $I_{\perp}$ are the emission intensities with polarizations in parallel and perpendicular directions of excitation polarization, respectively. In spherical quantum dot ensembles, the dipole moments are equal in all 3 dimensions $\left(\mu_{a}=\mu_{b}=\mu_{c}\right)$, the fluorescence anisotropy $r=0$, while the fluorescence anisotropy values are 0.4 and 0.1 for the ensembles of ideal nanorods $\left(\mu_{a}=\mu_{b} \ll \mu_{c}\right)$ and ideal nanodisks $\left(\mu_{a}=\mu_{b} \gg \mu_{c}\right)$, respectively, as presented in Fig. 1d and ESI. $\dagger$ Our DR solution shows the fluorescence anisotropy $r=0.12$, which corresponds to $\mu_{a}=\mu_{b}=0.46 \mu_{c}$. This anisotropy in the dipole moments will be utilized in our aligned DR structures.

CdSe/CdS DRs were dissolved in hexane $\left(5 \mathrm{mg} \mathrm{mL}^{-1}\right)$, and the solution was loaded into capillary tubes by capillary forces. The capillary tubes with a diameter of $300 \mu \mathrm{m}$ loaded with the DR solution were dried under reduced pressure or in air. As a result, DRs were deposited on the inner wall of the capillary tube. Two methods were employed to verify the optical anisotropy of the DR ensemble in the capillary tubes (Fig. 2). The first method, as illustrated in Fig. 2b, uses a linear polarized laser to excite the sample, and while tuning the polarization angle of the excitation beam with respect to the capillary tube's axis, the PL spectra of the DRs loaded in capillary are recorded. In the second approach, the sample was excited with a circularly polarized laser, and the polarization of PL was analyzed by varying the angle of the polarizer with respect to the capillary tube's axis before detection, as shown in Fig. 2c. These two methods are basically used to examine the polarization dependent absorption and emission of the CdSe/CdS DR ensemble in a capillary tube by varying the excitation and emission detection polarization angles, respectively. If the DRs are randomly oriented, i.e., their dipole moments are evenly distributed at all angles, the absorption and emission of the CdSe/CdS DR ensemble will not be polarization dependent. Fig. 2d presents the PL intensity of the DR ensemble as a function of excitation and emission detection polarization angles with respect to the capillary tube's axis corresponding to the two experiments. The results show that the preferable orientation for polarization of both absorption and emission is parallel to the capillary tube's axis. The same measurements were applied to the spherical nanocrystal ensemble in a capillary, as shown in Fig. 2e. Polarization dependent absorption and emission for spherical nanocrystals

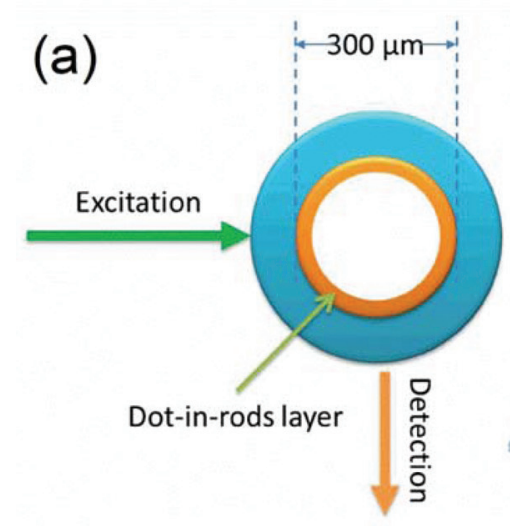

(d)

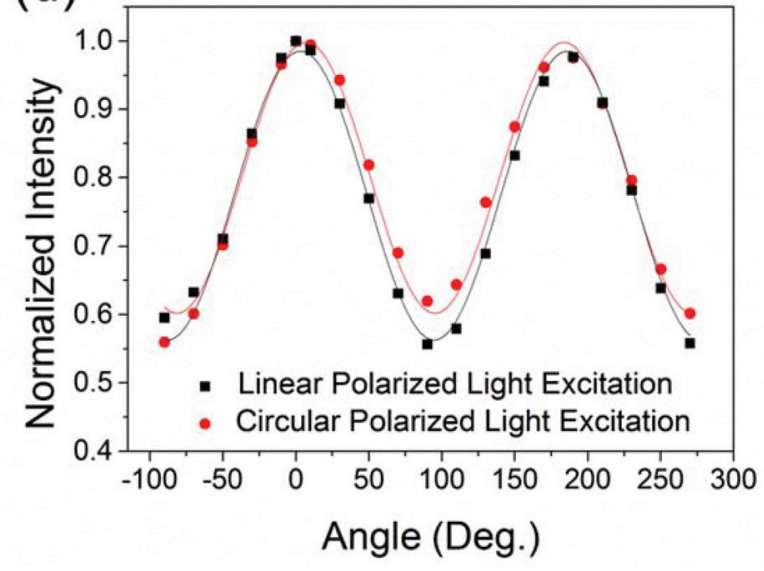

(b) (c) Capillary Tube with DRs

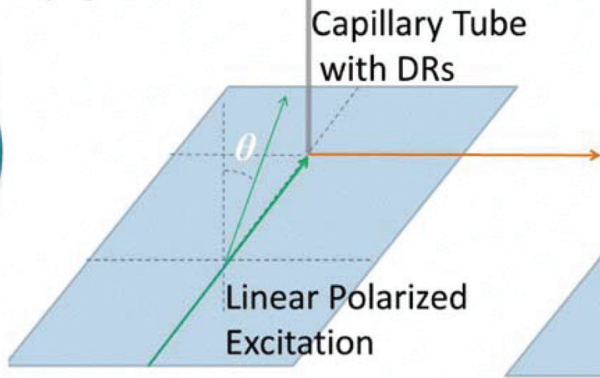

(e)

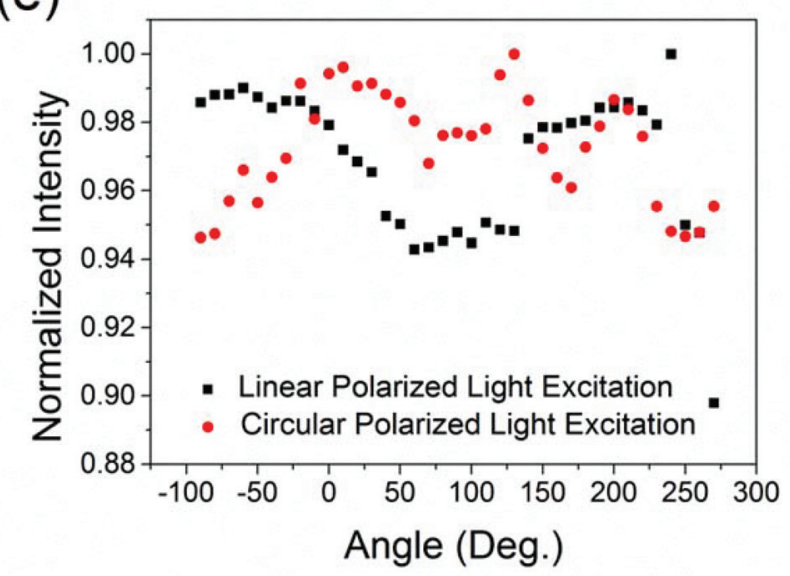

Fig. 2 Two approaches that were adopted for anisotropic absorption and emission measurements. (a) Cross section of a capillary tube with a dried $\mathrm{CdSe} / \mathrm{CdS}$ DR layer on its inner wall. (b) Excitation by linear polarized light for anisotropic absorption measurement: varying the polarization angle of excitation and monitoring the emission. (c) Excitation by circularly polarized light for anisotropic emission measurement: varying the polarization angle of the polarizer before the detector and monitoring the emission. (d, e) Normalized emission intensity of spontaneous emission as a function of the polarization angle with respect to the capillary tube in the two approaches for a capillary tube loaded with dried CdSe/CdS DRs (d) and CdSeS/ZnS spherical nanocrystals (e). 
dried in a capillary tube are negligible as compared to that of DRs. In addition, the anisotropic behavior was observed when the microcavity was broken by cracking the capillary tubes (Fig. S3†). Hence, any effect of the cylindrical microcavity on the anisotropic optical properties is ruled out, similarly to other reports about a large cylindrical microcavity. ${ }^{32}$ The data points were well fitted with the cosine-squared function of polarization angle (Fig. 2d): $\cos ^{2} \theta$, where $\theta$ is the angle between the capillary tube axis and the polarization of excitation or emission corresponding to our first or second method. The polarization ratio to characterize the degree of optical anisotropy of the DR ensemble in capillary tubes is defined as $p=\left(I_{\|}-I_{\perp}\right) /\left(I_{\|}+I_{\perp}\right)$, where $I_{\|}$and $I_{\perp}$ are the intensities of PL emission with polarization of excitation (or emission) parallel and perpendicular to the capillary tube, respectively. The polarization ratios calculated based on the fitting curves in Fig. 2d are 0.252 and 0.296 for emission and absorption, respectively. As shown above, individual CdSe/CdS DRs demonstrate the highest transition probability of absorption and emission along their $c$-axis; the high polarization ratios of the DR ensemble indicate that the DRs have a preferable orientation along the capillary tube. This is also statistically illustrated by scanning electron microscopy (SEM) images of the sample as shown in Fig. S4.†

Furthermore, the collective effects with stimulated emission of the aligned DRs in the capillary tubes were investigated. A more concentrated $\left(10 \mathrm{mg} \mathrm{mL}^{-1}\right) \mathrm{CdSe} / \mathrm{CdS}$ DRs solution was loaded into the capillary tube to deposit onto the inner wall of capillary tubes a preferable film for stimulated emission. The circularly polarized laser, generated from a frequency-doubled Nd:YAG pulsed laser at $532 \mathrm{~nm}$ with a repetition rate of $60 \mathrm{~Hz}$ and a pulse width of $1 \mathrm{~ns}$ and a quarter-wave plate, was used to excite the sample, and the emission anisotropy was examined by varying the polarizer before detection, as shown in Fig. 2c. Under low excitation energy, the emission from the sample was observed as spontaneous emission with a full width at half maximum (FWHM) of about $34 \mathrm{~nm}$, which is similar to those in the above experiments. Increasing the pump energy above the threshold, the emission spectrum became narrow and series of spiky peaks emerged, as shown in Fig. 3a. Closely investigating the lasing peaks (Fig. 3b), the peaks that are equally spaced with $\delta \lambda=0.21 \mathrm{~nm}$ can be resolved (Fig. 3c). The difference in $\delta \lambda$ between the TE mode (where the electric field is perpendicular to the tube axis, i.e., $-90^{\circ}$ or $90^{\circ}$ ) and the TM mode (where the electric field is parallel to the tube axis, i.e., $0^{\circ}$ ) is negligible. These well resolved lasing modes also suggest the optically smooth gain medium with negligible scattering, which is consistent with the SEM image of the CdSe/CdS DR layer on the inner wall of capillary tubes (Fig. S4d $\dagger$ ). As seen from a comparison given in ESI Fig. $S 5, \uparrow$ the mode spacing $(\delta \lambda)$ in the similar lasing structure with a smaller capillary tube diameter of $100 \mu \mathrm{m}$ is $\delta \lambda=$ $0.59 \mathrm{~nm}$, three times that at $300 \mu \mathrm{m}$ capillary tube diameter. This result confirms the WGMs in our cylindrical microcavities. For WGMs, $\delta \lambda=\lambda^{2} /(2 \pi n R)$, where the center emission wavelength is $\lambda=620 \mathrm{~nm}$ and the capillary tube radius is $R=$
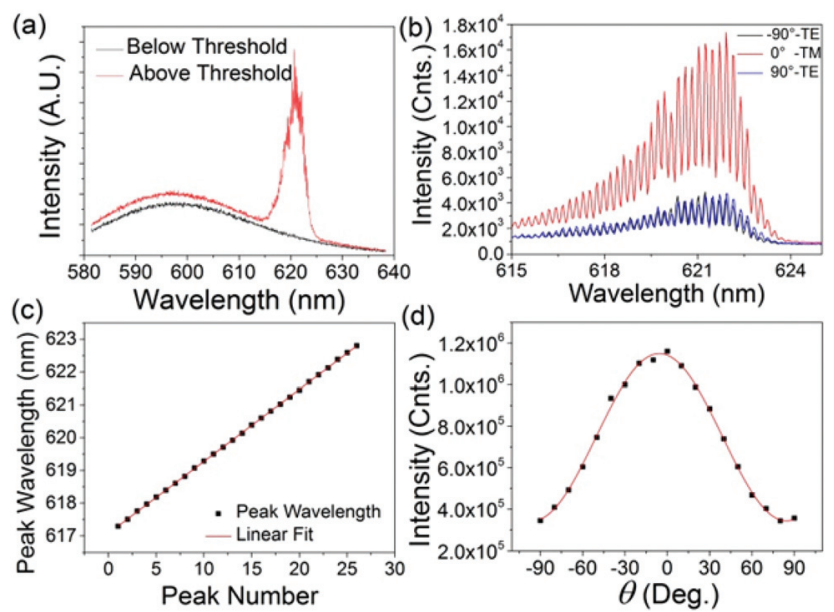

Fig. 3 Stimulated emission characterization from CdSe/CdS DRs in a capillary tube. (a) Emission of DRs in a capillary tube below and above the lasing threshold. (b) Spectra of lasing pumped by a circularly polarized laser from CdSe/CdS DRs in the capillary when the detected polarization is along the tube $\left(0^{\circ}, \mathrm{TM}\right.$ mode $)$ and perpendicular $\left(-90^{\circ}\right.$ and $90^{\circ}$, TE mode). (c) Plot of laser peaks versus the peak number and linear fit of the data. (d) Lasing intensity as a function of the detection angle with respect to the capillary tube, and fitted with $\cos ^{2} \theta$ function.

$150 \mu \mathrm{m}$, so the effective refractive index $n$ is about 1.92. Such a high refractive index suggests that the lasing modes had a large overlap with closely packed CdSe/CdS DR layers. The high refractive index contrast between the dense semiconductor nanocrystal layers and the glass as compared with that between the organic solvent and glass creates a desired laser configuration where the overlapping between optical modes and the gain medium can be maximized. Fig. $3 \mathrm{~b}$ also shows the different intensities of lasing spectra in TM and TE modes. As we can see, the lasing intensity in TM mode was much higher than that of TE mode lasing. The lasing intensity, integral of all the lasing peaks from 615 to $625 \mathrm{~nm}$, as a function of the polarization angle was measured and is shown in Fig. 3d under $7 \mu \mathrm{J}$ pump energy. As a comparison, the same measurement was conducted with spherical CdSeS/ZnS nanocrystals dried in capillary tubes, as shown in Fig. S6, $\uparrow$ and the emission normal to the capillary tube was slightly stronger than that along the capillary. This is due to the fact that the light which is polarized normal to the axis of the capillary tube is more likely to be transmitted out. The results show that the anisotropy of lasing emission in our laser configuration resulted from aligned DRs.

Fig. 4a presents the power transfer functions measured for TE and TM modes of our laser structure. The results are well consistent with "soft threshold" approximation, ${ }^{35}$ and the threshold was about $5.2 \mu \mathrm{J}$ for both TE and TM modes. As can be seen, initially the difference in lasing intensity between these two modes is small and then grows with increasing pumping energy. The evolution of the polarization ratio, $p$, as a function of pump energy is plotted in Fig. $4 \mathrm{~b}$. The polarization ratio of spontaneous emission started at about 0.05 and as the 


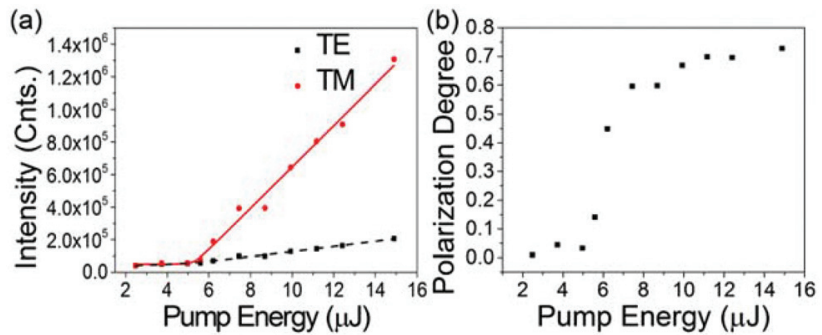

Fig. 4 Power transfer function of CdSe/CdS DRs in a capillary tube. (a) With increasing the pump energy, the evolution of lasing intensity, integral of all the lasing peaks, for TE and TM WGMs lasing, respectively. (d) Polarization ratio as a function of pump energy.

pumping energy increased, the polarization ratio built up and finally saturated at 0.72 . Here, we notice the polarization ratio reduction in spontaneous emission of this thicker DR film in comparison with that in Fig. 3. The increase of reabsorption in a thicker film could be the main reason for this polarization ratio reduction. However, a majority of $\mathrm{CdSe} / \mathrm{CdS}$ DRs are oriented along the capillary tube, creating a polarized gain medium with a higher gain coefficient for amplifying light with the electric vector along the tube. The small anisotropy of spontaneous emission is amplified in the polarized gain medium ${ }^{36}$ and resulted in a very large polarization ratio, $p=0.72$.

\section{Conclusions}

In summary, we demonstrated for the first time the utilization of the intrinsic optical anisotropy of CdSe/CdS DRs on a large scale for both spontaneous and stimulated emissions. Evaporating a CdSe/CdS DR solution inside a capillary tube leaves a thin film of aligned DRs on the inner wall of the tube. The polarization ratios of spontaneous absorption and emission for aligned DR films are 0.296 and 0.252 , respectively, while the WGM lasing resulting from the cylindrical microcavity has the maximum polarization ratio of 0.72 . Capillary tubes, after sealing in inert gas, also serve as oxygen and moisture free containers for CdSe/CdS DRs. Therefore, the lifetime and stability of these lasers can be much improved. Recently, LCD TVs were developed with nanocrystals in capillary tubes as the color convertors. ${ }^{37}$ Given that stimulated emission at red, green and blue colors can be observed from CdSe/CdS DRs, ${ }^{17}$ our approach with the capillary tubes demonstrating laser emission with a high polarization ratio could be used for novel display backlighting and laser lighting applications. ${ }^{38}$

\section{Experimental methods}

\section{Synthesis of CdSe/CdS dot-in-rods}

The synthesis approach is adopted from the literature. ${ }^{13}$ For CdSe seed synthesis, $3 \mathrm{~g}$ trioctylphosphine oxide (TOPO), $280 \mathrm{mg}$ octadecylphosphonic acid (ODPA), and $60 \mathrm{mg}$ cadmium oxide (CdO) were added into a $50 \mathrm{~mL}$ three-neck flask. Subsequently the mixture was degased at $150{ }^{\circ} \mathrm{C}$ for $2 \mathrm{~h}$. As the temperature was increased under an $\mathrm{Ar}$ atmosphere, $1.8 \mathrm{~mL}$ trioctylphosphine (TOP) was added after CdO dissolved completely. $58 \mathrm{mg}$ selenium in $0.36 \mathrm{~g}$ TOP was injected when the temperature reached $380^{\circ} \mathrm{C}$. Then the flask was removed from the heat mantle intermediately. As for the seeded nanorod growth, $3 \mathrm{~g}$ TOPO, $290 \mathrm{mg}$ ODPA, $80 \mathrm{mg}$ hexylphosphonic acid (HPA) and $86 \mathrm{mg}$ CdO were degased under vacuum for $2 \mathrm{~h}$ in a three-neck flask at $150^{\circ} \mathrm{C} .1 .8 \mathrm{~mL}$ TOP was added into the reaction vessel after the mixture became transparent, and then $200 \mu \mathrm{l}$ CdSe seeds (400 $\mu \mathrm{M}$ in TOP) together with $120 \mathrm{mg}$ sulfur in $1.5 \mathrm{~g}$ TOP were injected swiftly at $350{ }^{\circ} \mathrm{C}$. The as-synthesized CdSe/CdS DRs were cleaned with acetone and methanol, and then dissolved in hexane.

Wurtzite and zinc blende spherical nanocrystals were synthesized using one-pot ${ }^{39}$ and successive ion layer adsorption and reaction (SILAR) ${ }^{40}$ methods, respectively.

\section{Optical characterization}

Absorption and PL spectra were recorded using UV-1800 Shimadzu and RF-5301 PC. A continuous wave frequency doubled $\mathrm{Nd}$ :YAG laser at $532 \mathrm{~nm}$ was used for anisotropic optical property measurements of the DR solution and loaded in capillary tubes. For lasing characterization, the sample was pumped by a frequency doubled Nd:YAG pulsed laser at $532 \mathrm{~nm}$, whose repetition rate is $60 \mathrm{~Hz}$ and pulse width is $1 \mathrm{~ns}$. Meanwhile, the polarization response of the detector was measured and corrected, and the detection system shows very good polarization insensitivity (Fig. S7†).

\section{Acknowledgements}

The authors would like to thank the financial support from Singapore National Research Foundation under NRF-RF-2009-09 and NRF-CRP-6-2010-02 and the Science and Engineering Research Council, Agency for Science, Technology and Research (A*STAR) of Singapore (project no. 0921010057 and 112120 2009). The electron microscopy imaging was performed at the Facility for Analysis, Characterization, Testing and Simulation (FACTS) in Nanyang Technological University, Singapore. HVD also gratefully thanks TUBA and EURYI.

\section{Notes and references}

1 M. Bruchez, M. Moronne, P. Gin, S. Weiss and A. P. Alivisatos, Science, 1998, 281, 2013-2016.

2 J. Caruge, J. Halpert, V. Wood, V. Bulović and M. Bawendi, Nat. Photonics, 2008, 2, 247-250.

3 E. H. Sargent, Nat. Photonics, 2012, 6, 133-135.

4 V. Klimov, A. Mikhailovsky, S. Xu, A. Malko, J. Hollingsworth, C. Leatherdale, H.-J. Eisler and M. Bawendi, Science, 2000, 290, 314-317. 
5 J. Q. Grim, S. Christodoulou, F. Di Stasio, R. Krahne, R. Cingolani, L. Manna and I. Moreels, Nat. Nanotechnol., 2014, 9, 891-895.

6 D. V. Talapin, J.-S. Lee, M. V. Kovalenko and E. V. Shevchenko, Chem. Rev., 2009, 110, 389-458.

7 T. Erdem and H. V. Demir, Nat. Photonics, 2011, 5, 126126.

8 Y. V. Vandyshev, V. Dneprovskii and V. Klimov, JETP Lett., 1991, 53, 314.

9 V. Klimov, A. Mikhailovsky, D. McBranch, C. Leatherdale and M. Bawendi, Science, 2000, 287, 1011-1013.

10 F. García-Santamaría, Y. Chen, J. Vela, R. D. Schaller, J. A. Hollingsworth and V. I. Klimov, Nano Lett., 2009, 9, 3482-3488.

11 L.-W. Wang, M. Califano, A. Zunger and A. Franceschetti, Phys. Rev. Lett., 2003, 91, 056404.

12 V. I. Klimov, S. A. Ivanov, J. Nanda, M. Achermann, I. Bezel, J. A. McGuire and A. Piryatinski, Nature, 2007, 447, 441446.

13 L. Carbone, C. Nobile, M. De Giorgi, F. D. Sala, G. Morello, P. Pompa, M. Hytch, E. Snoeck, A. Fiore and I. R. Franchini, Nano Lett., 2007, 7, 2942-2950.

14 B. Guzelturk, Y. Kelestemur, M. Z. Akgul, V. K. Sharma and H. V. Demir, J. Phys. Chem. Lett., 2014, 5, 2214-2218.

15 B. Guzelturk, Y. Kelestemur, M. Olutas, S. Delikanli and H. V. Demir, ACS Nano, 2014, 8, 6599-6605.

16 Y. Kelestemur, A. F. Cihan, B. Güzeltürk and H. V. Demir, Nanoscale, 2014, 6, 8509-8514.

17 I. Moreels, G. Rainò, R. Gomes, Z. Hens, T. Stöferle and R. F. Mahrt, Adv. Mater., 2012, 24, OP231-OP235.

18 C. Grivas, C. Li, P. Andreakou, P. Wang, M. Ding, G. Brambilla, L. Manna and P. Lagoudakis, Nat. Commun., 2013, 4 .

19 M. Zavelani-Rossi, M. G. Lupo, F. Tassone, L. Manna and G. Lanzani, Nano Lett., 2010, 10, 3142-3150.

20 Y. Wang, V. D. Ta, Y. Gao, T. C. He, R. Chen, E. Mutlugun, H. V. Demir and H. D. Sun, Adv. Mater., 2014, 26, 29542961.

21 M. Zavelani-Rossi, M. G. Lupo, R. Krahne, L. Manna and G. Lanzani, Nanoscale, 2010, 2, 931-935.

22 F. Pisanello, G. Leménager, L. Martiradonna, L. Carbone, S. Vezzoli, P. Desfonds, P. D. Cozzoli, J.-P. Hermier, E. Giacobino, R. Cingolani, M. De Vittorio and A. Bramati, Adv. Mater., 2013, 25, 1974-1980.
23 J. Hu, L.-s. Li, W. Yang, L. Manna, L.-w. Wang and A. P. Alivisatos, Science, 2001, 292, 2060-2063.

24 J. S. Kamal, R. Gomes, Z. Hens, M. Karvar, K. Neyts, S. Compernolle and F. Vanhaecke, Phys. Rev. B: Condens. Matter, 2012, 85, 035126.

25 F. Pisanello, L. Martiradonna, G. Leménager, P. Spinicelli, A. Fiore, L. Manna, J.-P. Hermier, R. Cingolani, E. Giacobino and M. De Vittorio, Appl. Phys. Lett., 2010, 96, 033101.

26 A. Sitt, A. Salant, G. Menagen and U. Banin, Nano Lett., 2011, 11, 2054-2060.

27 A. Rizzo, C. Nobile, M. Mazzeo, M. D. Giorgi, A. Fiore, L. Carbone, R. Cingolani, L. Manna and G. Gigli, ACS Nano, 2009, 3, 1506-1512.

28 J. L. Baker, A. Widmer-Cooper, M. F. Toney, P. L. Geissler and A. P. Alivisatos, Nano Lett., 2009, 10, 195-201.

29 K. M. Ryan, A. Mastroianni, K. A. Stancil, H. Liu and A. Alivisatos, Nano Lett., 2006, 6, 1479-1482.

30 M. Kazes, D. Y. Lewis, Y. Ebenstein, T. Mokari and U. Banin, Adv. Mater., 2002, 14, 317-321.

31 Y. Wang, K. S. Leck, V. D. Ta, R. Chen, V. Nalla, Y. Gao, T. He, H. V. Demir and H. Sun, Adv. Mater., 2014, 27, 169175.

32 N. Frateschi, A. Kanjamala, A. Levi and T. Tanbun-Ek, Appl. Phys. Lett., 1995, 66, 1859-1861.

33 M. Grabolle, M. Spieles, V. Lesnyak, N. Gaponik, A. Eychmüller and U. Resch-Genger, Anal. Chem., 2009, 81, 6285-6294.

34 G. Xing, Y. Liao, X. Wu, S. Chakrabortty, X. Liu, E. K. Yeow, Y. Chan and T. C. Sum, ACS Nano, 2012, 6, 10835-10844.

35 J. Herrnsdorf, B. Guilhabert, Y. Chen, A. Kanibolotsky, A. Mackintosh, R. Pethrick, P. Skabara, E. Gu, N. Laurand and M. Dawson, Opt. Express, 2010, 18, 25535-25545.

36 B. E. A. Saleh and M. C. Teich, Fundamentals of Photonics, Wiley, 2013.

37 R. J. Nick, C. A. BREEN, C. M. Denton, S. Sadasivan and J. R. Linton, Google Patents, 2014.

38 A. Neumann, J. J. Wierer, W. Davis, Y. Ohno, S. R. Brueck and J. Y. Tsao, Opt. Express, 2011, 19, A982-A990.

39 W. K. Bae, J. Kwak, J. Lim, D. Lee, M. K. Nam, K. Char, C. Lee and S. Lee, Nano Lett., 2010, 10, 2368-2373.

40 O. Chen, J. Zhao, V. P. Chauhan, J. Cui, C. Wong, D. K. Harris, H. Wei, H.-S. Han, D. Fukumura and R. K. Jain, Nat. Mater., 2013, 12, 445-451. 